\title{
Phase I Clinical Trials in Oncology
}

\section{What Are the Steps in New Drug Development? \\ Preclinical phase}

This includes discovering or creating a potential drug, formulating it for appropriate delivery, in vitro studies, and animal studies.

\section{Traditional chemotherapy agents}

In earlier times, the candidate drugs (chemotherapy agents) were identified from natural compounds and may have been subsequently chemically modified to enhance their properties. These were tested in cell culture systems or in animal models and then subsequently formulated for human studies.

\section{"Targeted" agents}

The steps in the development of "targeted agents are: Target identification $\rightarrow$ Creating appropriate compounds to attack the target by chemical or bioengineering (monoclonal antibodies) $\rightarrow$ Formulation/in vitro studies/in vivo studies in animal models.

Additional preclinical studies may include assays to identify the target inhibition in animal models and pharmacokinetic and pharmacodynamic studies to know the doses that are able to inhibit the target.

Preclinical studies will establish the following:

1. The pharmacokinetics of the agent

2. The efficacy of the agent in vitro and in animal models

3. The feasibility of creating a formulation of the candidate agent

4. The toxicity and the "lethal" dose in mammalian species. "Lethal dose" may not always be achieved in case of "targeted" agents. $\mathrm{LD}_{50}$ is defined as the dose which kills $50 \%$ of the animals - This was an important dose level which helped to identify a starting point for phase I trials, especially for phase I trials of traditional chemotherapy agents

5. Target inhibition and bioassays to demonstrate the same.

\section{Clinical phase}

Once a drug has completed preclinical efficacy and toxicity studies, the sponsor decides to proceed with trials in humans and starts with phase I studies and subsequently II, III, and IV studies. Phase 0 refers to a specific type of "clinical" trial and is not a preclinical phase of the drug development. Before starting the clinical trials in humans, the sponsor has to file an "Investigational New Drug (IND)" application with the drug controller.

The definition of "IND" as per the CDSCO is "new chemical or biological entity or a product having a therapeutic indication, but which has never been tested before on human participants."

\section{What Is a Phase I Clinical Trial?}

Conventionally, these are "first-in-human" studies and would generally aim to establish the safety of an agent in human beings. Since conventional thinking is that higher the drug dose, more the cancer cell-kill, traditional drug development aimed to identify the highest dose of the drug that could be safely delivered.

The outcomes of interest from a phase I trial are:

1. Determine a dose that is appropriate for phase II and III testing (recommended phase II dose [RP2D])

2. Pharmacokinetics of the drug in human beings

3. Information about side effects in human beings

4. Early indications of activity and efficacy.

The information from animal studies was used for identifying the starting dose of an agent. This is conventionally one-tenth of the $\mathrm{LD}_{50}$ - the a dose is which was considered low enough, not to cause any harm.

\section{Some Important Terms}

\section{Dose-limiting toxicity}

Dose-limiting toxicity (DLT) is usually defined as any grade 3-4 non-hematological toxicity (except nausea and vomiting) or hematological toxicity lasting for $>7$ days. This definition traditionally refers to the first cycle of therapy. However, the definition may be altered based on the context and type of drugs and protocols specific. Example - in trials of "high-dose therapy" where stem cell rescue is planned, the hematological toxicity is usually not considered significant.

\section{Maximum tolerated dose}

Maximum tolerated dose (MTD) is the dose at which less than one-third of the participants develop DLT. It may be the same as the RP2D or may be different, especially for targeted agents where modeling based on pharmacodynamics or target inhibition is taken into consideration.

\section{Optimum biological dose}

Optimum biological dose (OBD) is the dose that produces a desired biological effect (e.g., target inhibition by pharmacodynamic correlative studies for "targeted" therapies, or modulation of a certain immune function identified by a specific assay). When conducting a phase I with a newer targeted or immunotherapy agent, DLT/MTD may not be achieved in the conventional sense. Hence, the OBD is defined to stop further dose escalations and conclude the trial. 


\section{Recommended phase II dose}

RP2D is again protocol specific. Conventionally, many phase I studies in the United States defined MTD as the RP2D. However, some other trials in Japan and Europe defined one dose level below the MTD as RP2D. The OBD may be considered as RP2D for targeted agents.

\section{What Is the Traditional " $3+3$ " Model of Drug Development?}

This is an empiric model designed to ensure the safety of participants. In this model, cohorts of three patients are treated at each dose level. If no DLT occurs, then the next three patients are doses at the dose level 2 and so on. If at any dose level, 1 of the three participants develops DLT, then 3 more are added at the same dose level. If 2 or more of the 6 develop DLT, then the dose level below will be considered as MTD. The MTD may be chosen as RP2D (in traditional chemotherapy agents) or a level lower than that may be chosen if it is felt that maximum target inhibition may be achieved.

\section{How Are Dose Levels Calculated in $3+3$ ?}

The first dose level is usually one-tenth of the $\mathrm{LD}_{50}$ as described above. The second level is twice the first, third level is $67 \%$ greater than the second, fourth is $50 \%$ greater than the third, fifth is $40 \%$ greater than the fourth, and subsequently, each level is $33 \%$ greater than the previous level. This method of escalation was empirically developed but continues to be used in practice, as it has been found to be associated with least chances of causing harmful side effects to participants. Since these numbers closely mimic the mathematical series, it has been called a modified Fibonacci method.

\section{How Do We Calculate Sample Size in a Phase I Trial?}

Sample size cannot be accurately calculated in a phase I, as we cannot be sure how many dose levels will be required to achieve MTD. However, a minimal sample size can be assumed as 12 patients (6 patients at level 1, and if DLT in 2 or more 6 additional patients at level "minus" 1). Most phase I trials with conventional agents realize MTD within 5-7 dose levels - hence, 30-50 patients are recruited. Some phase I trials may incorporate expansion cohorts and add patients at RP2D for specific tumor types to get early clues on efficacy. These can increase the size of phase I trials.

\section{Can a Drug Be Approved Based Only on Phase I Trial Data?}

It is rare but can happen when exceptional activity is demonstrated in rare conditions where no previous standard therapy exists. Example is the approval of crizotinib in ALK-positive lung cancer. Usually, regulatory agencies will require that the results be confirmed in a larger phase II or III trial.

\section{How Is a Phase I Trial of a "First-in-Human" Agent Different from Phase I Trials of New Combinations of Existing Agents?}

Phase I trials may also be conducted to identify the maximum doses of agents when combined together. For example, if someone wants to try a combination of eribulin and oxaliplatin - we do know the MTDs of each of these agents, but we do not know how much is to be used when in combination. Hence, the dose levels in this type of phase I trial will usually start with a lower than the usual dose of each of these agents and then escalate in a $3+3$ model. At each dose level there will be increase in doses of 1 or both agents as per the traditional 3+3 models (modified Fibonacci).

\section{What Are the Limitations of the $3+3$ Model?}

Although the $3+3$ model is considered as a safe model and studies have shown that most of the side effects of agents are actually well captured by the $3+3$ model, there are drawbacks.

1. It takes too many "levels" to find the MTD - we have to wait for all three patients at each dose level to complete at least one cycle before we can plan the next level. Hence, time is lost

2. Too many patients are treated at lower dose levels where the expectations of efficacy are minimal or nonexistent. Studies have shown that the chance of response in a phase I trial is around 5\%. Hence, majority of the participants do not benefit from the trial participation

3. For targeted agents, the MTD is not relevant as maximum target inhibition may be achieved at lower doses.

\section{Is the " $3+3$ " Model Still Valid in the Era of Targeted Treatment?}

Despite the problems detailed above, $3+3$ model is still a popular design practiced for majority of newer agents in oncology. This is because of the overall safety of this approach. Further, the newer designs (detailed below), though attractive in theory, have not really helped to speed up the process in practice. For example, in models such as Bayesian designs, real-time input of data is required with continuous feedback from the statistician, making it impractical to use in many situations.

\section{What Are the Other Models of Phase I Trials?}

1. Accelerated titration design: in this model, intrapatient patient dose escalation is permitted from cycle to cycle. Doses are escalated in consequtive cycles for the same patient till DLT occurs. Trials with these designs are able to reduce the number of patients treated at subtherapeutic doses but are not able to reduce the 
overall time duration required to complete the study. This kind of model is suitable for rare cancer subtypes.

2. Continuous reassessment model (CRM): This uses a Bayesian "prior distribution" to determine the steepness of the dose-toxicity curve. This requires updating of information after each patient is treated. Then, the curve is readjusted and dose level for the next patient is determined

3. For targeted agents - model by Korn et al. Initial accelerated phase - one patient in each dose level until a biological effect is seen. Then cohorts of 3-6 in each level. If 5-6 responses are seen at a particular level that is determined as biologically active and trial is closed

4. Rolling 6 design is a method which was proposed for pediatric phase I and recently has become popular. Pediatric phase I trials often focus on "refining" the MTD, which is already known from adult phase I trials (not to actually find MTD for a new drug). Here, the dose window of operation is often narrower, and this model allows faster completion than a traditional $3+3$. Here, 2-6 patients are accrued simultaneously in a particular dose level. The level itself is determined by number already enrolled, number of DLTs, and number at risk of DLTs.

\section{Can a Phase I Trial Used to Assess Efficacy?}

Even though the primary aim of a phase I trial is not to assess efficacy, most phase I studies report preliminary signals of efficacy. Phase I trials that are histological subtype agnostic can give early indicators as to which cancers are likely to benefit. This may guide the design of phase II studies with that agent. From the patient's perspective, efficacy is of primary concern when they agree to participate in a phase I trial though the informed consent process clearly states that they may not get any benefit. Overall, the chance of responses in phase I trials is quite low (response rates 5\%-10\%).

\section{What Are the Rules in India for the Conduct of Phase I Trials?}

Although phase I trials are allowed by law, there are certain specific rules. They should be carried out by investigators trained in the field and having necessary facilities to closely observe the patients. In general, phase I trial (first-in-human) is permitted for new drug entities developed in India. For molecules developed abroad, first-in-human studies are permitted only if the study has already enrolled patients in some other country.

\section{What are Phase Ib and Phase I/II Trials?}

In phase $\mathrm{Ib}$ trials, there is simultaneous recruitment of patients at multiple dose levels. It is a modified trial design aimed at accelerating the process of finding the RP2D. It is also useful for dose-finding studies using combinations of agents
Phase I/II trials involve a design of phase I to identify the RP2D, and then, the same trial continues to recruit patients in the phase II portion at the RP2D. The patients already recruited at RP2D level in the phase I cohort will also be contribute to the required sample size for the phase II, thus speeding up the drug development process.

\section{How are Phase I Trials of Agents used for High-Dose Therapy Different?}

The main difference here is that hematological toxicities are not considered dose limiting as they are salvaged with the stem cell infusion.

\section{What Are the Minimum Facilities Needed for Conducting a Phase 1 Trial?}

- Clinicians familiar with the conducting phase I trials including ways of recording toxicities, and looking out for unexpected toxicities

- Statisticians with the ability to manage more complicated designs such as the CRM

- Trial coordinators/research nurses who are trained to communicate with these patients

- Laboratory facilities for handling multiple patient samples for pharmacokinetic work including facilities such as HPLC. Added capacity for pharmacodynamic assessment (target inhibition and immunological assays) would be desirable

- Administrative capability to handle massive amounts of paper work required for these kinds of studies to fulfill regulatory requirements

- Large patient pool is again desirable as many patients will have to be screened to find eligible participants.

\section{What Are the Special Challenges in Performing} a Phase I Trial in Pediatric Patients?

Pediatric trials often start after completion of adult trials and focus more on refining the MTD. A fair idea about the toxicity profile of an agent is already available when starting a pediatric trial. Pediatric cancers are rarer and very few targeted agents are currently approved. Within the "pediatric' population, there is wide heterogeneity by size and age. Traditional $3+3$ models have been used for a long time, but they have problem of long accrual times because we have to suspend enrolment after each block of three have completed at least one cycle and assessment. Hence, newer models such as rolling 6 have been developed. As for all clinical trials involving minors, consent is obtained from parents/guardians and must include a witness signature. Assent must be obtained from adolescent participants.

Financial support and sponsorship

Nil. 


\section{Conflicts of interest}

There are no conflicts of interest.

\section{Prasanth Ganesan}

Department of Medical Oncology, JIPMER, Puducherry, India

Address for correspondence: Dr. Prasanth Ganesan, Department of Medical Oncology, JIPMER, Puducherry, India.

E-mail: p.ganesan@jipmer.edu.in

Submitted: 09-Jan-2020

Revised: 26-Apr-2020

Accepted: 04-Jun-2020

Published: 31-Dec-2020
This is an open access journal, and articles are distributed under the terms of the Creative Commons Attribution-NonCommercial-ShareAlike 4.0 License, which allows others to remix, tweak, and build upon the work non-commercially, as long as appropriate credit is given and the new creations are licensed under the identical terms.

\begin{tabular}{|l|l|}
\hline \multicolumn{2}{|c|}{ Access this article online } \\
\hline Quick Response Code: & Website: \\
& www.ijmpo.org \\
\cline { 2 - 2 } & DOI: \\
\hline
\end{tabular}

How to cite this article: Ganesan P. Phase I clinical trials in oncology. Indian J Med Paediatr Oncol 2020;41:885-8. 